\title{
Neue Ordnungsökonomik. Zur Aktualität eines kontextualen Forschungsprogramms: Kommentar zum Beitrag von Stefan Kolev, Nils Goldschmidt und Joachim Zweynert
}

\author{
Björn Frank
}

Online publiziert: 1. April 2019

(C) List-Gesellschaft e.V. 2019

\section{Worum geht es eigentlich?}

Programme sind grundsätzlich auf die Zukunft ausgerichtet, wie zum Beispiel Kinoprogramme oder Artenschutzprogramme. Forschungsprogramme sind da keine Ausnahme, und ihre Kernelemente sind m.E.:

a) Ein Verständnis darüber, welches die ungelösten der vorrangigen Probleme einer Wissenschaft sind (oder die vorrangigen der ungelösten), und

b) ein Verständnis darüber, mit welchen Methoden diese Probleme anzugehen sind.

Natürlich besteht die Versuchung, in der Darstellung eines Forschungsprogramms auch die vergangenen Erfolge ihrer Vertreter aufzuführen (wie das auch bei Wahlprogrammen der Fall ist), aber Kolev, Goldschmidt und Zweynert (2019) (Kolev et al. im Folgenden) erliegen ihr nicht. Darauf deutet nicht nur das Adjektiv „,neu“ in der „Neuen Ordnungsökonomik“ hin, sondern auch Passagen wie der Einstieg zu Abschn. 4, in dem mit erfrischender Offenheit der ,offensichtlichen Bedeutungsverlust der Ordnungsökonomik“ u.a. ihren Vertretern angelastet wird, die sich lange darauf beschränkt hätten, ,die Güte des ursprünglichen Programms zu preisen“ Chapeau! Wenn es Kolev et al. um die Aktualität der Ordnungsökonomik geht, dann wollen sie also, denke ich, nicht zeigen, wie stark alte Erkenntnisse in neuem Licht noch glänzen. Meine Frage an ihren Beitrag ist daher, ob sie folgende Punkte machen können:

\footnotetext{
This commentary article refers to the article available online at https://doi.org/10.1007/s41025-01900155-2. An author's reply to this commentary article is available online at https://doi.org/10.1007/ s41025-019-00158-z.
}

\footnotetext{
B. Frank $(\bowtie)$

Universität Kassel, Kassel, Deutschland

E-Mail: frank@uni-kassel.de
} 
c) Aktuelle Probleme sind solche, zu deren Lösung insbesondere die Ordnungsökonomik berufen ist, und/oder

d) Der methodische Werkzeugkasten der Ordnungsökonomik hat sich jüngst derart weiterentwickelt - oder er ist gerade dabei, sich derart weiterzuentwickeln -, dass die Ordnungsökonomik Probleme besser angehen kann als sie das früher konnte, und besser als andere Zweige der Ökonomik.

Im Folgenden gehe ich auf Kolev et al.'s Diskussion von Forschungsfragen und Methoden ein, wobei ich nicht sehe, dass ihnen Fehler im engere Sinne nachzuweisen sind. Ich glaube allerdings, dass arg viele Fragen offen geblieben sind, deren Klärung ich vielleicht provozieren kann. Jedenfalls scheint es mir typisch für die Ordnungsökonomik zu sein, dass das hohe Abstraktionsniveau ihres Beitrags viele Desiderata bedingt.

\section{Desiderata}

\subsection{Forschungsfragen}

In Abschn. 1 nennen Kolev et al. drei Phänomene, die sich allein durch das Standardinstrumentarium nicht erklären ließen:

- Donald Trump

- Die Fragilität der Europäischen Union

- Die Euro-Krise

Das erste Beispiel trifft zweifellos zu. In normalen Zeiten würde die bloße Nennung des Namens eines gewählten Regierungschefs noch nicht hinreichen, um ein Problem zu beschreiben, aber gegenwärtig ist die Krisensituation mit „Trump“ präzise benannt. Zugleich unterstützt das Beispiel Trump gut eine zentrale Forderung von Kolev et al., nämlich ,kontextuale“ Ökonomik zu betreiben, was hier m. E. konkret heißt, beispielsweise folgende Fragen aus der Schnittmenge von Ökonomik, Soziologie und Politikwissenschaft zu beantworten: Wieso haben amerikanische Wähler gegen ihre eigenen (ökonomischen) Interessen gewählt? (Die Vermutung, dass das so ist, stellt den ,,isolierenden“ rational choice-Ansatz jedenfalls in Frage.) Ist das vorhersagbares Verhalten, und für welche Länder oder Situationen sagen wir dieses vorher? ${ }^{1}$

Die Euro-Krise ist dagegen orthodox erklärbar; ich vermute, dass die Mehrzahl der Ökonomen, die sich sogar selbst dem Mainstream zuordnen würden, sich über die europaweiten Wirkungen der aktuellen Krise in Italien nicht wundert. Während die ökonomische Krise der Gemeinschaftswährung wohl unstrittig ist, halte ich es nicht für ausgemacht, dass damit auch ein Problem der orthodoxen Ökonomik identifiziert wurde. Ganz im Gegenteil, Kolev et al. bleiben den Nachweis für ihre Behauptung schuldig, dass die ,Entwicklung der vergangenen Jahre (...) sich als ein Prozess der kumulativen Ordnungserschütterungen kennzeichnen“ lasse. Das ist

\footnotetext{
1 Eine neue ökonomische Studie dazu ist Algan et al. (2017).
} 
sicher nicht nur auf die Eurokrise bezogen, aber egal, welchen konkreten Fall wir betrachten, wie ließen sich denn überhaupt „kumulativen Ordnungserschütterungen“ diagnostizieren? Welches wären die Methoden?

\section{2,Methoden der Ordnungsökonomik“}

Mit den Augen eines „orthodoxen Verhaltensökonomen“ (Kollegenzitat) gelesen kommt nur eine Methode bei Kolev et al. zum Zuge, das sind Feldstudien am Beispiel der Arbeiten von Elinor Ostrom. Ja, so kann man Ordnungsökonomik betreiben: Beobachten, unter welchen Bedingungen und wie es Gesellschaften gelingt, mit Widersprüchen zwischen individueller und kollektiver Rationalität fertig zu werden und so beispielweise die massive Übernutzung natürlicher Ressourcen (die ,Tragödie der Allmende“) abzuwenden. Zwar kann man mit guten Gründen der Methode der Fallstudie eine häufigere Verwendung in der Ökonomik wünschen (Swann 2006, Kap. 17), aber das kann ja nicht alles sein.

Ökonomen und andere Sozialwissenschaftler, die sich dafür interessieren, welche Institutionen funktionieren und welche Regeln kontraproduktiv sind, bedienen sich seit einigen Jahren experimenteller Methoden. Experimente sind ,isolierend“, weil sie den Einfluss der Treatmentvariablen zu isolieren imstande sind, das ist ihre Stärke und nicht ihre Schwäche. Sie sind aber auch „kontextuell“, weil sie Treatmentvariablen untersuchen können, über die Hypothesen typischerweise nur mit Hilfe der Nachbardisziplinen entwickelt werden. Kontextuell sind sie auch, weil man neuerdings versucht, sie unter sonst gleichen Bedingungen in unterschiedlichen Kontexten durchzuführen; exemplarisch seien nur zwei Studien genannt, die übrigens beide nicht in ökonomischen Zeitschriften veröffentlicht wurden. Henrich et al. (2005) zeigen, wie sich Unterschiede in der ökonomischen Organisation indigener Gesellschaften im Verhalten ihrer Mitglieder in einfachen ökonomischen Experimenten widerspiegelt. Muthukrishna et al. (2017) variieren Elemente ökonomischer Ordnungen in einem public good game mit (möglicher) Korruption.

Ob das nun Ordnungsökonomik ist, wird mir auch nach wiederholter Lektüre von Kolev et al. nicht klar.

Eine anderer - kleiner aber wachsender ${ }^{2}$ - Literaturzweig sind kliometrisch-ökonomische Studien. Mit quantitativen Methoden untersuchen sie beispielsweise das Beharrungsvermögen informeller Institutionen. Becker et al. (2016) nutzen die Tatsache, dass fünf Länder heute teilweise innerhalb und teilweise außerhalb der Grenzen des Habsburger Reiches liegen, um zu zeigen, dass sich auch nach 100 Jahren noch ordnungspolitisch relevante (positive) Effekte der vergleichsweise guten Habsburger Verwaltung zeigen: Höheres Vertrauen in Gerichte und Polizei, weniger Korruption. Das mag verblüffend sein, aber weltweit gibt es Befunde dieser Art - um nur noch ein weiteres Beispiel zu nennen: Das 1812 aufgegebene System der Zwangsarbeit in einem Teil der Silberminen Perus und Boliviens hat bis heute anhaltende negative lokale Effekte; die (ordnungsökonomischen?) Mechanismen dieser Persistenz versucht Dell (2010) aufzuklären.

\footnotetext{
2 Vgl. Abramitzky (2015).
} 
Immer ist dabei Ökonometrie im Spiel. Die Schätzung von Koeffizienten einer Regressionsgleichung kann man per se ,isolierend“ finden, man kann diese Studien aber auch als solche sehen, die helfen, den Kontext zu verstehen; auf welche Seite sich Kolev et al. schlagen, wüsste man gern.

Ich hätte allerdings wenig Hoffnung, mit experimentellen oder kliometrischen Methoden den speziellen Punkt der „,kumulativen Ordnungserschütterungen“ zu erhellen, andere Methoden liegen in diesem Fall näher. Zwei Vorbemerkungen zum Begriff:

Wenn - erstens - mein Eindruck zutrifft, dass die „alten“ und wohl auch die „,neuen“ Ordnungsökonomen eine stärkere Präferenz als andere Ökonomen haben, auf Deutsch statt auf Englisch zu kommunizieren, dann liegt das vermutlich an der Bedeutung suggestiver Begriffe wie „kumulative Ordnungserschütterung“, die nicht isolierend sind, weil alles Mögliche mitschwingt und mitschwingen soll; dies ist in einer Fremdsprache schwieriger durch Begutachtungsprozesse zu bringen. Leichter fiele das mit präziser Hypothesenentwicklung und statistischen Hypothesentests.

Nehmen wir - zweitens - einfach an, mit kumulativer Ordnungserschütterung sei nicht einfach ein wiederholter Bruch von Regeln (wie zum Beispiel den Bestimmungen des Stabilitäts- und Wachstumspaktes der EU) gemeint, sondern die Wahrnehmung der Mitglieder einer Gesellschaft, dass die bestehende Ordnung bedroht ist oder ihren Wert verliert, sei es durch politischen Zerstörungswillen einzelner Mächtiger, sei es durch eine Dynamik, die aus der Interaktion vieler Einzelinteressen entsteht.

Warum sollte man sich dann mit dem Eindruck begnügen, den Ordnungsökonomen vom Eindruck der Bürger haben? Interdisziplinäre empirische Forschungen darüber, wie Nichtökonomen volkswirtschaftliche Zusammenhänge wahrnehmen, gibt es bereits, teilweise unter dem Schlagwort „Folk Economics“ (Rubin 2003; Leiser und Shemesh 2018)3. Kann das eine Methode der Ordnungsökonomik sein? Muss es das sein, wenn zu einer kontextualen Ökonomik nicht nur die Sicht der Ökonomen auf die Wirtschaft und Gesellschaft gehört, sondern auch die der Bürger?

\section{Der Bürger}

Kolev et al. sähen gern, wenn die Neue Ordnungsökonomik ein „besserer Berater des Bürgers mitten in den Transformationsprozessen der global-digitalen Ordnungen von Wirtschaft und Gesellschaft" wäre. Aus meiner Sicht ist das noch ein weiter, weiter Weg, von dem Kolev et al. kaum einen Millimeter zurücklegen, dabei ist ihr Papier aufschlussreich und lesenswert, aber das, was mir daran richtig gut gefällt, ist gleichzeitig das, was „den Bürger“ weder berührt noch interessiert:

\footnotetext{
3 Ökonomische Phänomene werden manchmal direkt wahrgenommen (der Supermarkt in der Nachbarschaft schließt), meist aber gefiltert durch die Medien. Die selektive Wahrnehmung von Informationen durch Medienkonsumenten und das Interesse von Medien, Schwerpunkte in Hinblick auf die eigene Klientel selektiv zu setzen oder zu verzerren, war für zwei Jahrzehnte Gegenstand ökonomischer Diskussionen (z. B. DellaVigna und Kaplan 2007; McCarthy und Dolfsma 2014) - dezidiert ordnungsökonomische Beiträge zu dieser Diskussion würden mich interessieren.
} 
Erstens rekonstruieren sie sehr schön, wie die Entwicklung der ökonomischen Theorie vom ordnungspolitischen Umfeld und der Entwicklung der Wirtschaftssysteme beeinflusst wurde. Das erscheint sehr plausibel und anregend, wenn man erst einmal darauf gestoßen wird. Zweitens finde ich es sehr hilfreich für das Nachdenken über Forschungsstile, wenn sie treffende Bezeichnungen bekommen und treffend voneinander abgegrenzt werden, all dies gelingt Kolev et al. mit ihrem Gegensatzpaar isolierende versus kontextuale Ökonomik, die sie übrigens auch beide fair charakterisieren. Drittens - wie schon erwähnt - gefällt mir der nüchterne Umgang mit der ,,alten“ Ordnungsökonomik.

Den Bürger aber spricht das kaum an. Meine abschließende Frage ist, ob es vorstellbar ist, so über Ordnungsökonomik zu schreiben, dass das breit rezipiert wird. Das wäre sicher im Sinne von Kolev et al., die die Attraktivität der Ordnungsökonomik für das 21. Jahrhundert darin sehen, dass sie traditionell ,,isolierende und kontextuelle Fragen zueinander in Beziehung zu setzen" versucht, also in einer Vermittlungsleistung, was an anderer Stelle noch deutlicher wird: „Will Ökonomik interdisziplinär anschlussfähig sein und politische Konsequenzen zeitigen, bedarf es des verbalen Arguments und der Sprachfähigkeit von Ökonomen. “ Das ist zweifellos richtig. Wie sind nun die Aussichten auf einen spürbaren ordnungsökonomischen Beitrag hierzu?

Karen Horn hatte das Privileg, einige Jahre in der FAZ auf ihre Weise viel über Ordnungsökonomik schreiben zu dürfen, aber der letzte ordnungsökonomische Bestseller war wohl Hayeks The Road to Serfdom (1944). Der prototypische populärwissenschaftliche Besteller der zeitgenössischen Ökonomik ist allerdings Freakonomics (mit dem - m. E. besseren - Nachfolgeband SuperFreakonomics; Levitt und Dubner 2005, 2009). Diesem Projekt gingen zahlreiche wissenschaftlich anspruchsvolle, originelle Forschungsarbeiten voraus, das eigentliche Thema von Freakonomics lautet „Die quasiexperimentelle Bestimmung von Kausalität bei der mikroökonomischen Analyse von traditionell nichtökonomischen Phänomenen“, und wenn man daraus mit Glück und Geschick ${ }^{4}$ einen Bestseller machen kann, dann vielleicht auch aus ordnungsökonomischer Forschung. Das hielte ich für ein ehrenwertes Bestreben, allerdings noch nicht für ein Forschungsprogramm.

\section{Literatur}

Abramitzky, R. (2015). Economics and the modern economic historian. Journal of Economic History, 75, $1240-1251$.

Algan, Y., Guriev, S., Papaioannou, E., \& Passari, E. (2017). The European trust crisis and the rise of populism. Brookings Papers on Economic Activity, 2017(2), 309-382.

Becker, S. O., Boeckh, K., Hainz, C., \& Woessmann, L. (2016). The empire is dead, long live the empire! Long-run persistence of trust and corruption in the bureaucracy. Economic Journal, 126, 40-74.

Dell, M. (2010). The persistent effects of Peru's mining Mita. Econometrica, 78, 1863-1903.

DellaVigna, S., \& Kaplan, E. (2007). The fox news effect: Media bias and voting. Quarterly Journal of Economics, 122, 1187-1234.

Frank, B. (2012). Economic page turners. Journal of Economic Methodology, 19, 317-327.

Hayek, F. A. (1944). The road to serfdom. London: Routledge.

Henrich, J., Boyd, R., Bowles, S., Camerer, C., Fehr, E., Gintis, H., McElreath, R., Alvard, M., Barr, A., Ensminger, J., Smith, N.H., Hill, K., Gil-White, F., Gurven, M., Marlowe, F. W., Patton, J. Q.,

\footnotetext{
4 Vgl. dazu Frank (2012).
} 
\& Tracer, D. (2005). "Economic man" in cross-cultural perspective: Behavioral experiments in 15 small-scale societies. Behavioral and Brain Sciences, 28, 795-815.

Kolev, S., Goldschmidt, N., Zweynert, J. (2019), Neue Ordnungsökonomik. Zur Aktualität eines kontextualen Forschungsprogramms. List Forum für Wirtschafts- und Finanzpolitik. https://doi.org/10.1007/ s41025-019-00155-2.

Leiser, D., \& Shemesh, Y. (2018). How we misunderstand economics and why it matters. The psychology of bias, distortion and conspiracy. London, New York: Routledge.

Levitt, S. D., \& Dubner, S. J. (2005). Freakonomics. New York: HarperCollins.

Levitt, S. D., \& Dubner, S. J. (2009). SuperFreakonomics. London: Allen Lane.

McCarthy, K. J., \& Dolfsma, W. (2014). Neutral media? Evidence of media bias and its economic impact. Review of Social Economy, 72, 42-54.

Muthukrishna, M., Francois, P., Pourahmadi, S., \& Henrich, J. (2017). Corrupting cooperation and how anticorruption strategies may backfire. Nature Human Behaviour, 1(7), 138. https://doi.org/10.1038/ s41562-017-0138.

Rubin, P.H. (2003). Folk economics. Southern Economic Journal, 70, 157-171.

Swann, G. M. P. (2006). Putting econometrics in its place. Cheltenham \& Northampton: Elgar. 